\title{
Vorgeformte winkelstabile Rekonstruktionsplatten zur Überbrückung von Defektzonen im Unterkiefer
}

\author{
Florian Andreas Probst, Sebastian Schiel, Carl-Peter Cornelius, Gerson Mast, \\ Michael Ehrenfeld, Michael Ermer, Marc Christian Metzger, Ralf Gutwald, \\ Rainer Schmelzeisen
}

\begin{abstract}
Zusammenfassung
MatrixMANDIBLE Preformed Reconstruction Plates (MMPRP) sind neuartige, anatomisch vorgeformte Rekonstruktionsplatten zur winkelstabilen Überbrückung unterschiedlicher Defekttypen im anterolateralen Unterkieferbereich. Sie eignen sich sowohl für eine Insertion über den klassischen extraoralen Zugang als auch für die transorale Applikation. Erste klinische Erfahrungen deuten darauf hin, dass das Risiko von Ermüdungsbrüchen und die Operationszeit aufgrund der Präformierung der Platten sinken. Insgesamt sind MMPRP als ein geeignetes Standardsystem für die winkelstabile und lasttragende Osteosynthese im Bereich des Unterkiefers zu bewerten. Dies gilt für eine Vielzahl von Indikationen aus der Tumorchirurgie, Traumatologie sowie beim Management von Osteonekrosen.
\end{abstract}

Preformed Angle-Stable Reconstruction Plates for Bridging Defect Zones in the Lower Jaw

MatrixMANDIBLE preformed reconstruction plates (MMPRP) are novel anatomically preshaped load-bearing macroplates, which provide for anglestable bridging of different mandibular defect types. They are suitable for a standardised extraoral approach as well as for a transoral approach. First clinical experiences indicate that the risk for fatigue fractures may be lowered. Additionally, operation time seems to be decreased. All in all, MMPRP present as a useful standard device for mandibular reconstructions in a wide scope of indications, ranging from tumour through trauma to osteonecrosis.

\section{Einleitung}

Rekonstruktionsplatten spielen eine wichtige Rolle bei der Versorgung ausgedehnter Defekte des Unterkiefers. Die erste Generation dieser groß dimensionierten Plattensysteme wurde Mitte der 1970er-Jahre eingeführt und ersetzte alte Verfahren wie beispielsweise Drahttechniken [18]. Während die ersten Rekonstruktionsplatten noch aus Edelstahl bestanden, wurde Titan bald zum Material der Wahl. Rekonstruktionsplatten gewährleisten eine lasttragende Osteosynthese im Unterkieferbereich und stabilisieren sowohl verbleibende Knochenstrukturen als auch indirekt das umgebende Weichgewebe [6]. Anwen-

OP-JOURNAL 2012; 28: 188-192

(c) Georg Thieme Verlag KG Stuttgart · New York DOI http://dx.doi.org/10.1055/s-0032-1315360 dungsgebiete für die Platten reichen von Unterkieferresektionen im Rahmen onkochirurgischer Eingriffe über die Versorgung von Trümmerfrakturen bis zur Überbrückung defektähnlicher Bereiche, wie sie bei Bisphosphonat- oder Osteoradionekrosen anzutreffen sind [4,6,22].

Unterkieferrekonstruktionsplatten wurden mit den technischen Neuerungen im Laufe der Jahre immer wieder modifiziert. Der bedeutendste Fortschritt ist dabei der Einführung rigider winkelstabiler Verankerungssysteme zu verdanken, welche zu einer Verbesserung der Biomechanik und der Handhabung führten. Als erstes System winkelstabiler Rekonstruktionsplatten war in den 1980erJahren das sog. „Titanium-coated hollow screw and reconstruction plating system" (THORP; Synthes Maxillofacial, Paoli, PA, USA) verfügbar [17]. Dieses System beruht darauf, dass die enossalen
Schraubenimplantate im Kopfbereich durch eine Expansionsschraube in den Plattenlöchern verbolzt werden und somit die gewünschte Winkelstabilität entsteht. Ende der 1990er-Jahre etablierten sich schließlich sog. Locking-Rekonstruktionsplatten-Systeme [5,7]. LockingSchrauben können durch ein zusätzliches Gewinde im Kopfbereich in den korrespondierend gefrästen Gewinden der Plattenlöcher verankert werden. Durch die Schraubenverriegelung wird eine lasttragende Osteosynthese im Sinne des Fixateur-interne-Prinzips erreicht. Dabei werden die einwirkenden Kräfte direkt vom Knochen auf die verriegelten Schrauben und folglich auf die Platte übertragen, ohne auf den Defektbereich einzuwirken. Die Platte übernimmt alle einwirkenden Kaukräfte und überbrückt somit den Defektbereich biomechanisch. Im Gegensatz zu Adaptationsplatten ist es nicht zwingend erforderlich, die Platte absolut präzise an die Unterkiefergeometrie anzupassen. Aufgrund der biomechanischen Vorteile und der universellen Verwendung haben sich diese winkelstabilen Platten etabliert $[5,7,20]$.

\section{Typische Probleme}

Die Verwendung von Rekonstruktionsplatten ist häufig mit Komplikationen wie Ermüdungsbrüchen, Platten- oder Schraubenlockerungen, Plattenexpositionen durch die Schleimhaut in die Mundhöhle oder durch die äußere Haut sowie Fistelbildungen verbunden.

Als Risikofaktoren sind neben einer Radiotherapie als wesentlicher Faktor auch Tumorrezidive sowie Unterernährung relevant $[5,9,13]$. Mit Blick auf die Literatur ist interessanterweise feststellbar, dass die Fortschritte im Plattendesign nicht zu einer Reduktion dieser typischen Probleme geführt haben [9]. Darüber hinaus bleibt die umständliche und zeitintensive Handhabung mit umfangreichen intraoperativen Biegemanövern ein immer wieder vorgebrachter Kritik- 
punkt bei der Verwendung von Unterkiefermakroplatten. Nicht zuletzt trägt mehrmaliges Biegen zu einer erhöhten Wahrscheinlichkeit für Ermüdungsbrüche bei.

\section{Warum vorgeformte Platten?}

Die genannten Nachteile brachten die Idee zu einem neuartigen Plattendesign, bei dem die Grundform der Platte mit einem besonders stabilen Zentralabschnitt bereits vorgebogen ist und nur noch in geringem Umfang an die Kontur des Unterkiefers angepasst werden muss. Auf der Basis dieses Konzepts wurden die sog. MatrixMANDIBLE Preformed Reconstruction Plates (MMPRPs) von der Firma Synthes ${ }^{\circledR}$ (Paoli, USA) entwickelt. Diese anatomisch vorgeformten, lasttragenden Osteosyntheseplatten ahmen die durchschnittliche Kontur der Lateralfläche der anterolateralen Mandibula nach. Die Plattenform basiert auf einer dreidimensionalen morphometrischen Analyse von über 2000 CT-basierten Datensätzen. Anhand multivariater statistischer Methoden hat sich eine Gruppierung in 3 Größen und die jeweilige Errechnung von Durchschnittsformen als sinnvoll erwiesen. Damit ist es möglich, eine große Mehrheit der natürlich auftretenden Formgegebenheiten des Unterkiefers zu repräsentieren [14].

\section{Indikationen/Defekttyp}

Die Größe und der lasttragende Charakter der MMPRP prädestinieren die Platte für die Überbrückung unterschiedlichster Defekttypen bzw. defektähnlicher Situationen und erlauben einen Einsatz in der ganzen Bandbreite der rekonstruktiven kraniomaxillofazialen Chirurgie (Tab. 1).

Dies betrifft klassischerweise Segmentdefekte nach Unterkieferkontinuitätsresektion aus onkologischen Gründen. Aber auch weniger ausgedehnte Defekte (marginaler Defekt bzw. defektähnliche Situation), wie sie nach Unterkieferkastenresektionen, nach Entfernung benigner Tumoren oder nach Abtragung osteonekrotischen Knochens vorzufinden sind, können durch MMPRP überbrückt werden. Ebenso bieten sie eine wertvolle Option bei Trümmerfrakturen, Frakturen des atrophen Unterkiefers und zur Stabilisierung von Pseudarthrosen. Gerade bei kleinerer Defektausdehnung und defektähnlichen Zonen erleichtert das vorgeformte Plattendesign die Möglichkeit zur transoralen Plattenapplikation.

Tab. 1 Indikationen sowie typische korrespondierende Defektsituationen und Zugangsmöglichkeiten für die lasttragende Osteosynthese mit MatrixMANDIBLE Preformed Reconstruction Plates (MMPRP).

\begin{tabular}{lll}
\hline Indikation & Defekttyp & Zugang \\
\hline Malignom (PLE-Ca, OS, Mx) & segmental/marginal & extraoral \\
\hline benigner Tumor & segmental/marginal & extraoral/transoral \\
\hline Trümmerfrakur & defektähnlich & extraoral/transoral \\
\hline Pseudarthrose & defektähnlich & extraoral/transoral \\
\hline Osteonekrose (ORN, BRONJ) & segmental/marginal/defektähnlich & extraoral/transoral
\end{tabular}

PLE-Ca $=$ Plattenepithelkarzinom, $\mathrm{OS}=$ Osteosarkom, $\mathrm{Mx}=$ Metastasierung, $\mathrm{ORN}=$ Osteoradionekrose, $\mathrm{BRON}]=$ bisphosphonatassoziierte Osteonekrose des Kiefers

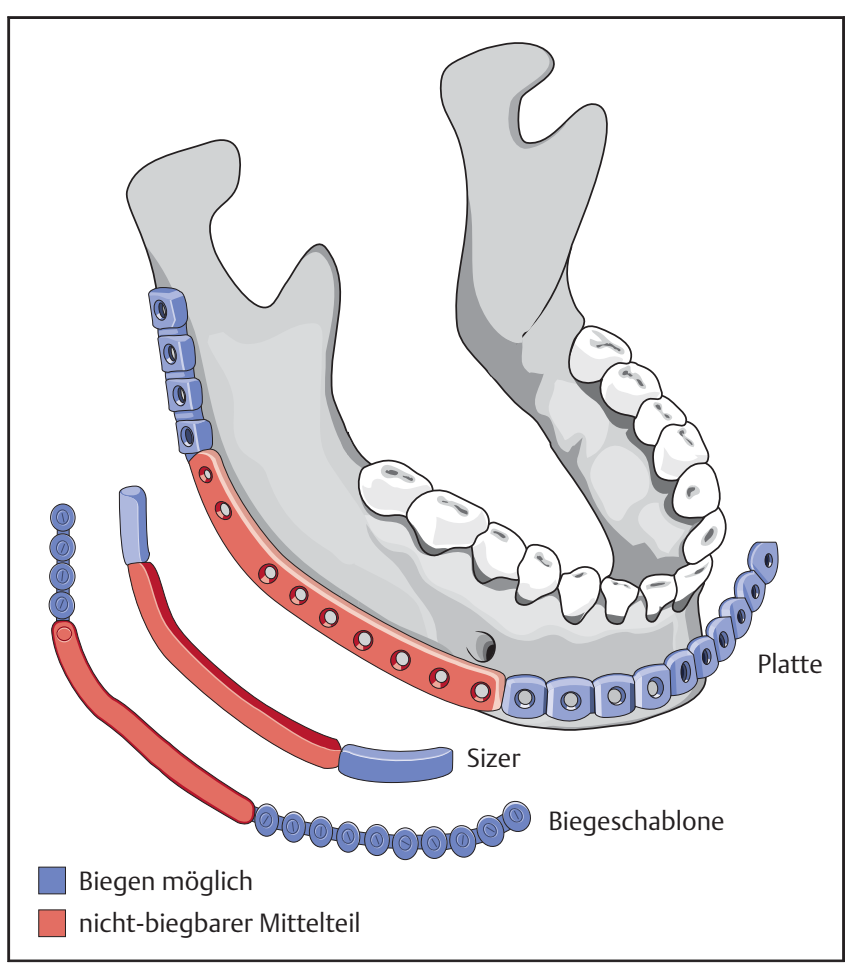

Abb. 1 MatrixMANDIBLE Preformed Reconstruction Plate (MMPRP) an die laterale Fläche eines Unterkiefers angelegt. Mitte: korrespondierender Sizer. Unten: Biegeschablone. Nicht biegbarer Mittelteil jeweils rot dargestellt. Biegbarer proximaler und distaler Anteil jeweils blau dargestellt.

\section{Operationstechnik}

MatrixMANDIBLE Preformed Reconstruction Plates (MMPRP) stehen in 3 Größen („small“, „medium“, „large“) zur Verfügung. Sie bestehen aus einem mittleren, nicht biegbaren Zentralabschnitt ohne Plattenkerben, welcher der Form der Lateralfläche des Unterkiefers entspricht, und aus zwei für Biegemanöver vorgesehenen Anteilen proximal und distal (Abb. 1).

Die bestgeeignete Plattengröße wird durch Anlegen von Metall-Sizern an die anterolaterale Oberfläche der Mandibula bestimmt. Eine geringfügige Modifizierung der gewählten Plattenlänge kann später noch durch Erweiterung oder Verringerung der Krümmung über die Fläche im Kinnbereich erreicht werden. Als nächster Schritt wird mithilfe von dünnen Biegeschablonen die Kontur der Platte im proximalen und distalen Anteil bestimmt. Zunächst wird der zentrale Abschnitt der Schablone an der Innenseite der Rekonstruktionsplatte oder des Sizers angelegt und in die übereinstimmende Krümmung gebracht. Anschließend wird die Schablone am Unterkiefer angelegt und im Kinn- und Ramusbereich manuell auf der Knochenoberfäche adaptiert, um sie abzuformen. Falls notwendig, wird die Schablone gekürzt. Dabei ist zu beachten, dass im proximalen und distalen Abschnitt mindestens 3-4 Schraubenlöcher zur Verfügung stehen. Dementsprechend wird nun die Rekonstruktionsplatte mit den Schneideinstrumenten eingekürzt und die am Unterkiefer abgegriffene Schablonenform mit Biegezangen und Schränkeisen auf die 


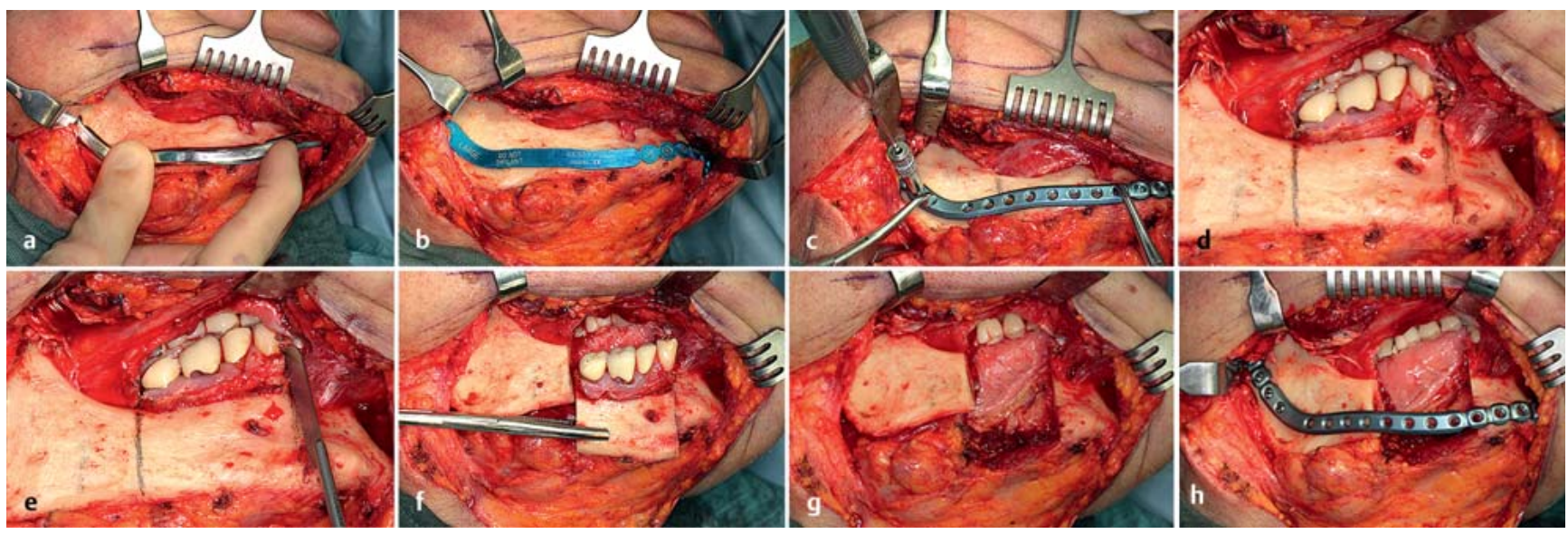

Abb. $\mathbf{2 a}$ bis $\mathbf{h}$ Schilderung des Resektionsmodus bei einem Patienten mit ameloblastischem Karzinom im Bereich des Unterkiefers rechts. a Bestimmung der Plattengröße mit dem Sizer. b Ankonturieren der Biegeschablone. c Fixierung der Platte mit Locking-Schrauben. d Einzeichnen der Resektionsgrenzen. e Unterkiefersegmentresektion mit oszillierender Stichsäge. f Entnommenes Segment im Bereich des Korpus rechts. g Segmentaler Defekt. h Definitive Applikation der Platte.
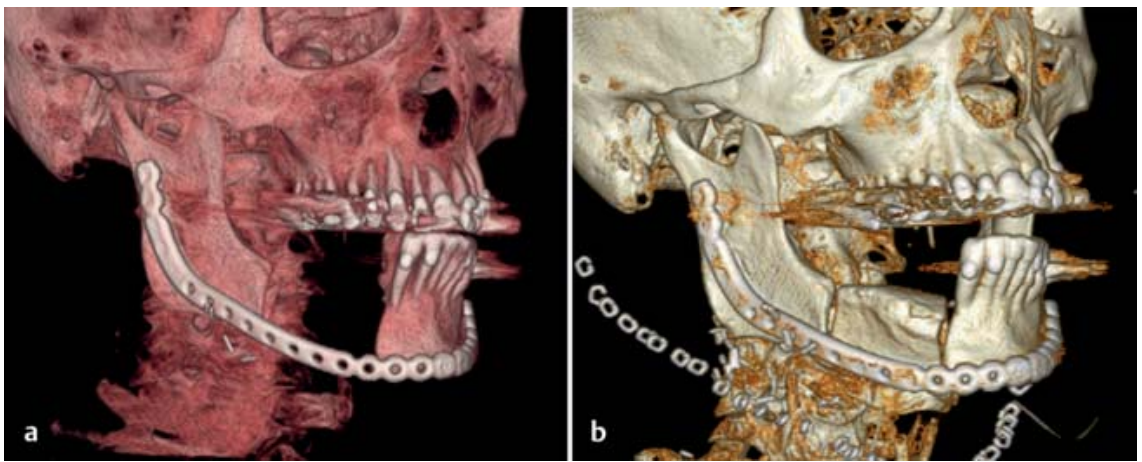

Abb. 3 3-D-Rekonstruktionen von CTs des Patienten aus Abb. 2 nach zweizeitigem Vorgehen zur autologen Unterkieferrekonstruktion. Oben segmentale Unterkieferresektion rechts bei ameloblastischem Karzinom und Stand-alone-MMPRP. Unten: sekundäre Rekonstruktion mittels mikrovaskulär reanastomosiertem Beckenkammtransplantat.

Platte übertragen. Die Biegung erfolgt inner- und außerhalb der Plattenebene. Dazu ist die Biegezange mit je einem speziellen Schloss zum „In-Plane“- und zum „Out-of-Plane“-Bending ausgestattet. Begonnen wird proximal oder distal jeweils mit dem Schraubenloch, das sich direkt am nicht formbaren Plattenabschnitt befindet. Mit den Schränkeisen kann die Platte in eine Drehstellung um die Längsachse (Torquierung) gebracht werden. Nach Abschluss der Konturierung wird die Platte auf der Lateralfläche des Unterkiefers positioniert und mit Haltezangen temporär fixiert. In die Platte können Locking-Schrauben mit 2,4 mm oder 2,9 mm Durchmesser inseriert werden. Zum Anbringen der Bohrlöcher stehen 3 Arten an Bohrhülsen (Gewindebohrbüchse, Doppelbohrbüchse oder transbukkales Instrumentarium) zur Verfügung. Das 1. Bohrloch wird unmittelbar proximal der Defektregion im posterioren Segment angelegt. Bohr- löcher sollten generell möglichst senkrecht zur Plattenebene angelegt werden, um später Winkelstabilität zu erreichen. Nach dem Abmessen der Schraubenlänge mit der Tiefenlehre erfolgt die Insertion einer Verriegelungs-(Locking-) Schraube. Das 2. Bohrloch wird unmittelbar distal der Defektregion im anterioren Segment angelegt und mit einer Schraube belegt. Die restlichen Schrauben werden wechselseitig im proximalen und distalen Unterkiefersegment eingebracht.

\section{Resektionsmodus}

Im Falle einer segmentalen oder kastenförmigen Resektion hat sich folgendes Vorgehen als praktikabel erwiesen (Abb. 2). Nach Festlegung und gegebenenfalls Anzeichnen der Resektionsränder erfolgt zunächst die bereits beschriebene Applikation (inkl. Fixierung) der Platte. Nun werden die Schrauben wieder entfernt und entsprechend ihrer
Position in einem dafür bestimmten Schrauben-Caddy verwahrt. Das vorgesehene Unterkiefersegment wird reseziert und die Platte anschließend in der ursprünglichen Position erneut fixiert. In den Defekt kann entweder ein temporärer alloplastischer Knochenersatz (z.B. Medpor) oder ein avaskuläres bzw. mikrovaskulär reanastomosiertes Knochentransplantat eingebracht werden (Abb. 3). Alternativ bleibt es bei der alleinigen Defektüberbrückung mit der Platte als sog. „Stand-alone“-Rekonstruktion.

\section{Transorale Applikation}

Während Unterkieferrekonstruktionsplatten in aller Regel nur über einen externen submandibulären Zugang eingebracht werden, lassen sich MMPRP aufgrund ihrer vorgeformten Geometrie auf vergleichsweise einfache Weise auch transoral applizieren (Abb.4). Nach vestibulärer Eröffnung der Mukoperiosts entlang des Alveolarfortsatzes zwischen der Vorderkante des aufsteigenden Astes und der Kinnregion, auf einem Level oberhalb des $\mathrm{N}$. mentalis werden die Lateralfläche des ipsilateralen Ramus, Kieferwinkels, Korpus und die Symphysenregion subperiostal dargestellt. Um sicher oberhalb des $\mathrm{N}$. mentalis zu bleiben, wird die Schnittführung in der Prämolarenregion direkt an der mukogingivalen Grenzlinie oder bei Zahnlosigkeit krestal vorgenommen. Das Nervenbündel wird behutsam subperiostal abgelöst und von der Unterlage mobilisiert, womit ein Pfad für die Platte geschaffen wird, welcher unterhalb des Nervens basal am Unterkieferrand entlanggeht. Im Anschluss an die Frakturreposition oder vor einer 

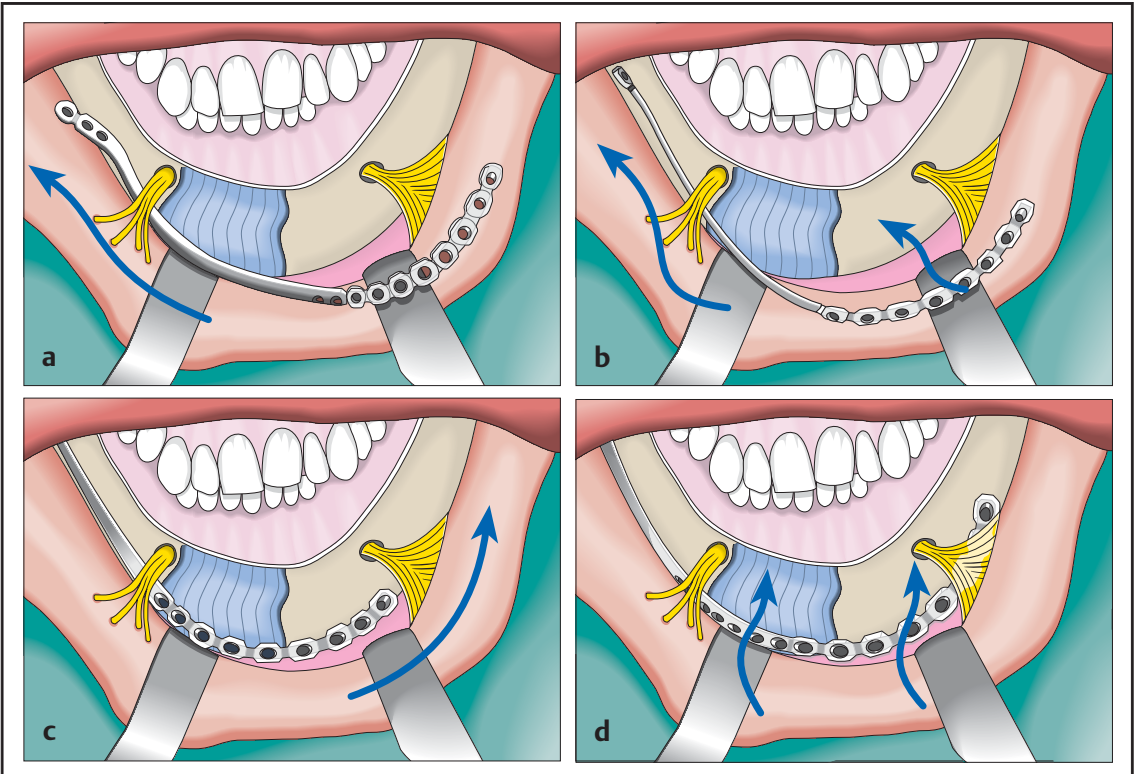

Abb. 4a bis d Defektregion im Bereich des Korpus sowie paramedian rechts (markierter Bereich). Transorale Applikation der Platte bis in den Unterkieferkorpus der Gegenseite. a Initiales Einfädeln der Platte unterhalb des ipsilateralen Nervenbündels nach posterior. b Weiterführen der Platte nach posterior, unter Rotation des hinteren Plattenendes nach kranial, bis sich das vordere Ende etwa in der Mittellinie befindet. c Vorschieben der Platte auf die Gegenseite. d Durchführen des vorderen Plattenendes unter dem kontralateralen Nervenbündel, um die Platte in ihre Endposition zu bringen.

Knochenresektion wird die geeignete Plattengröße bestimmt, indem der bestpassende Sizer in den tunnelierten Gewebebereich unterhalb des N. mentalis eingeschoben wird. Insbesondere bei Trümmerfrakturen ist es hilfreich, die kleineren Fragmente nach anatomischer Reposition zunächst mit Miniplatten zu verbinden, bevor zur lasttragenden Stabilisierung eine MMPRP eingesetzt wird. Genau wie der Sizer wird die Biegeschablone unterhalb des Nervenbündels inseriert, um die Form und Krümmung der Unterkieferlateralfläche im Ramus und Kinnbereich abzugreifen. Auch eine transorale Applikation der Platte bis in den Unterkieferkorpus der Gegenseite ist möglich. Dazu muss die vestibuläre/krestale Schnittführung hufeisenförmig nach kontralateral erweitert und die Nervenaustrittregion beider Nn. mentales vorbereitet werden. Die Schablone bzw. später die Platte werden unterhalb des ipsilateralen Nervens nach posterior eingefädelt und mit dem hinteren Ende soweit nach kranial rotiert, bis sich das vordere Ende etwa in der Mittellinie befindet. Dann wird die Schablone/ Platte auf die Gegenseite vorgeschoben und das vordere Ende unter dem kontralateralen $\mathrm{N}$. mentalis durchgeführt, um die Platte in ihre beidseitige Endposition zu bringen. Im Ramusbereich erweist sich die Fixierung der Platte mit einem
Winkelbohrer/-schraubendrehersystem anstelle der transbukkalen Applikation als sinnvoll.

\section{Eigene Erfahrungen und Diskussion}

\section{Komplikationen}

Plattenfrakturen gehören bisher zu den typischen Komplikationen bei Verwendung von Unterkieferrekonstruktionsplatten und werden mit einer Frequenz von ca. $3-10 \%$ beobachtet $[8,10,12,19-$ 21]. Als wesentliche Einflussgröße gelten dabei aufwändige Biegeprozeduren, welche residualen Stress im Bereich der Platte induzieren und letztlich Ermüdungsbrüche begünstigen [13].

Infolge der anatomischen Präformierung der MMPRP können exzessive Biegeprozeduren verhindert werden, womit das Risiko für Ermüdungsbrüchen sinkt.

Als Vorteil ist ebenso das durchgängig glatte, nicht biegbare Mittelstück aufzuführen, welches keine Kanten oder Ecken aufweist. Denn gerade im Bereich von Einkerbungen der Plattenstruktur kommt es zur Stresskonzentration. Während es in unserem eigenen Krankengut bei 70 Patienten mit inserierter MMPRP bisher zu keinem Plattenbruch kam, traten andere typische, mit Rekonstruk- tionsplatten assoziierte Komplikationen mit der gleichen Frequenz auf, die im Schrifttum angegeben wird [16]. Dies betrifft sowohl Plattenexpositionen durch Haut oder Schleimhaut und Platten-/Schraubenlockerungen als auch die Ausbildung von Fisteln [1-3, 5,9,12,15]. Der Hauptrisikofaktor für Plattenexpositionen und Fistelbildung ist nach Literatur und eigener Erfahrung die Radiotherapie $[3,5,12,15,16]$. Veränderungen im Plattendesign scheinen hinsichtlich Expositionsrate und orokutanen Fistelbildungen keinen wesentlichen Fortschritt erbringen zu können, was sich ebenfalls in unserem Krankengut widerspiegelt.

\section{Passgenauigkeit}

In einer eigenen Fallserie war bei $71 \mathrm{~Pa}$ tienten nur in einem Fall die Passung des nicht biegbaren Mittelteils der Platte derart ungenügend, dass die präformierte Platte nicht verwendet werden konnte. In den meisten Fällen war die Passgenauigkeit sehr zufriedenstellend. Für das stets notwendige Anbiegen des proximalen und distalen Anteils der Platte wurde beachtlich weniger Zeit beansprucht als beim Anbiegen konventioneller Rekonstruktionsplatten [16]. Allerdings bleibt zu erwähnen, dass die statistische Analyse, die dem Plattendesign zugrunde liegt, eigentlich einer Kurve und nicht einer Fläche entspricht. Somit sind Torsionen der Unterkiefergeometrie nicht berücksichtigt. Dies mag einer der Gründe dafür sein, dass die Passgenauigkeit zwar als zufriedenstellend, jedoch nicht immer als sehr gut einzuschätzen ist.

Letztlich verzeihen MMPRP, wenn sie als winkelstabile Platten zum Tragen kommen, in einem gewissen Maße Ungenauigkeiten, da sie im Sinne eines Fixateur interne fungieren und nicht wie Adaptationsplatten exakt anzupassen sind.

Als Alternative zu MMPRP soll noch die Möglichkeit aufgeführt werden, konventionelle Rekonstruktionsplatten präoperativ an einem Stereolithografiemodell anzubiegen [11]. Diese indirekte Methode zur Präformierung hat das Potenzial, intraoperative Anpassungsprozeduren noch weiter zu minimieren oder sogar unnötig zu machen. Jedoch sind hierfür hohe Kosten bspw. durch Modellherstellung aufzurechnen. 


\section{Transorale Applikation}

Aufgrund ihrer Präformierung vereinfachen MMPRP die transorale Applikation erheblich.

Als Option kann diese Variante insbesondere beim Vorliegen von Trümmerfrakturen, Frakturen beim atrophen Unterkiefer sowie bei Pseudarthrosen oder Osteonekrosen in Erwägung gezogen werden. Beim Vorliegen von Osteonekrosen mit intraoral exponiertem Knochen kann vermutet werden, dass durch die Vermeidung eines extraoralen Zugangs das Risiko für Wundheilungsstörungen mit Ausbildung einer intra-/extraoralen Fistel sinkt. Weitere Vorteile einer transoralen Vorgehensweise liegen in der Vermeidung störender äußerer Narben, in der Umgehung des Läsionsrisikos für den $\mathrm{R}$. marginalis mandibulae des $\mathrm{N}$. facialis sowie einer verminderten Operationszeit. Das Einfädeln unter den N. mentalis bringt sicherlich ebenfalls ein Schädigungspotenzial mit sich, das aber dank der Präformierung, des glatt durchgängigen Plattenmittelstücks ohne Einkerbungen und bei behutsamem Vorgehen als gering eingeschätzt werden darf. Insgesamt steht nun eine praktikable Möglichkeit zur Verfügung, groß dimensionierte, lasttragende Unterkieferosteosyntheseplatten transoral zu applizieren.

\section{Fazit}

Anhand der bisherigen Erfahrungen mit der MatrixMANDIBLE Preformed Reconstruction Plate (MMPRP) können folgende Schlussfolgerungen gezogen werden. MMPRP minimieren das Risiko für Ermüdungsbrüche. Bezüglich anderer typischer Komplikationen wie Plattenexposition oder Fistelbildung ergeben sich jedoch keine Vorteile. Die Handhabung der Platten ist komfortabel und geht mit einer verkürzten Operationszeit einher. Zudem vereinfacht die Präformierung eine transorale Applikation in erheblicher Weise. Alles in allem steht unserer Meinung nach mit der MMPRP ein winkelstabiles Osteosynthesesystem zur Verfügung, das sich vom bisherigen Niveau lasttragender Plattensysteme zur Versorgung von Defektsituationen im Unterkiefer qualitativ abhebt.

\section{Literatur}

${ }^{1}$ Alonso del Hoyo J, Fernandez Sanroman J, Rubio Bueno $P$ et al. Primary mandibular reconstruction with bridging plates. J Craniomaxillofac Surg 1994; 22: 43-48

2 Arden RL, Rachel JD, Marks $S$ et al. Volumelength impact of lateral jaw resections on complication rates. Arch Otolaryngol Head Neck Surg 1999; 125: 68-72

3 Cordeiro P. Hidalgo D. Soft tissue coverage of mandibular reconstruction plates. Head Neck 1994; 16: 112-115

${ }^{4}$ Ellis E, Muniz O, Anand K. Treatment considerations for comminuted mandibular fractures. J Oral Maxillofac Surg 2003; 61: 861-870

5 Gellrich NC, Suarez-Cunqueiro MM, Otero-Cepeda XL et al. Comparative study of locking plates in mandibular reconstruction after ablative tumor surgery: THORP versus UniLOCK system. J Oral Maxillofac Surg 2004; 62: 186-193

${ }^{6}$ Goh BT, Lee S, Tideman H et al. Mandibular reconstruction in adults: a review. Int J Oral Maxillofac Surg 2008; 37: 597-605

${ }^{7}$ Herford AS, Ellis E. Use of a locking reconstruction bone plate/screw system for mandibular surgery. J Oral Maxillofac Surg 1998; 56: 1261-1265

${ }^{8}$ Katakura A, Shibahara T, Noma H et al. Material analysis of AO plate fracture cases. J Oral Maxillofac Surg 2004; 62: 348-358

${ }^{9}$ Klotch DW, Gal TJ, Gal RL. Assessment of plate use for mandibular reconstruction: has changing technology made a difference? Otolaryngol Head Neck Surg 1999; 121: 388-392

10 Lavertu P, Wanamaker IR, Bold EL et al. The AO system for primary mandibular reconstruction. Am J Surg 1994; 168: 503-507

11 Lethaus B, Poort L, Bockmann R et al. Additive manufacturing for microvascular reconstruction of the mandible in 20 patients. J Craniomaxillofac Surg 2012; 40: 43-46

${ }^{12}$ Lopez R, Dekeister C, Sleiman Z et al. Mandibular reconstruction using the titanium functionally dynamic bridging plate system: a retrospective study of 34 cases. J Oral Maxillofac Surg 2004; 62: 421-426

${ }^{13}$ Martola M, Lindqvist C, Hanninen H et al. Fracture of titanium plates used for mandibular reconstruction following ablative tumor surgery. J Biomed Mater Res B Appl Biomater 2007; 80: 345-352

14 Metzger MC, Vogel M, Hohlweg-Majert B et al. Anatomical shape analysis of the mandible in Caucasian and Chinese for the production of preformed mandible reconstruction plates. J Craniomaxillofac Surg 2011; 39: 393-400

15 Nicholson RE, Schuller DE, Forrest LA et al. Factors involved in long- and short-term mandibular plate exposure. Arch Otolaryngol Head Neck Surg 1997; 123: 217-222

16 Probst FA, Mast G, Ermer $M$ et al. MatrixMANDIBLE preformed reconstruction plates - a two-year two-institution experience in 71 patients. J Oral Maxillofac Surg 2012 Aug 3 [Epub ahead of print]

17 Raveh J, Stich H, Sutter F et al. Use of the titanium-coated hollow screw and reconstruction plate system in bridging of lower jaw defects. J Oral Maxillofac Surg 1984; 42: 281294

18 Schmoker R, Spiessl B, Mathys R. A total mandibular Plate to Bridge large Defects of the Mandible. In: Spiessl B, ed. New Concepts in maxillofacial Bone Surgery. Berlin: Springer; 1976: 381-398

${ }^{19}$ Schöning H, Emshoff R. Primary temporary AO plate reconstruction of the mandible. Oral Surg Oral Med Oral Pathol Oral Radiol Endod 1998; 86: 667-672

${ }^{20}$ Schupp W, Arzdorf M, Linke B. Biomechanical testing of different osteosynthesis systems for segmental resection of the mandible. J Oral Maxillofac Surg 2007; 65: 924-930

${ }^{21}$ Shibahara T, Noma H, Furuya Y. Fracture of mandibular reconstruction plates used after tumor resection. J Oral Maxillofac Surg 2002; 60: 182-185

${ }^{22}$ Wong RC, Tideman H, Kin $L$ et al. Biomechanics of mandibular reconstruction: a review. Int J Oral Maxillofac Surg 2010; 39: 313-319

\section{Dr. med. dent. Florian Andreas}

\section{Probst}

Assistenzarzt

Dr. med. Dr. med. dent.

Sebastian Schiel

Oberarzt

Prof. Dr. med. Dr. med. dent. Carl-Peter Cornelius

Oberarzt

Dr. med. Dr. med. dent. Gerson Mast

Leitender Oberarzt

Prof. Dr. med. Dr. med. dent.

Michael Ehrenfeld

Direktor der Klinik

Klinik und Poliklinik für Mund-,

Kiefer- und Gesichtschirurgie

Klinikum der Universität München

Ludwig-Maximilians-Universität

München

Lindwurmstraße 2a

80337 München

florian.probst@med.uni-muenchen.de

Dr. med. Dr. med. dent.

Michael Ermer

Assistenzarzt

Priv.-Doz. Dr. med. Dr. med. dent.

Marc Christian Metzger

Oberarzt

Prof. Dr. med. Dr. med. dent.

Ralf Gutwald

Leitender Oberarzt

Prof. Dr. med. Dr. med. dent.

Rainer Schmelzeisen

Direktor der Klinik

Klinik und Poliklinik für Mund-,

Kiefer- und Gesichtschirurgie

Universitätsklinikum Freiburg

Albert-Ludwigs-Universität Freiburg

Hugstetterstraße 55

79106 Freiburg 